\title{
PREVALENCE OF ANXIETY, DEPRESSION AND STRESS AMONG MEDICAL STUDENTS, AND ASSOCIATED FACTORS
}

\author{
By \\ Gabal HA, Wahdan MM and Gamal Eldin DA \\ Department of Community, Environmental and Occupational Medicine, Faculty of Medicine, Ain \\ Shams University, Egypt. \\ Corresponding author: Gabal HA.Email: babosha2008@med.asu.edu.eg
}

DOI: 10.21608/ejom.2021.72495.1232

Submit Date: 2021-04-21Ｒevise Date: 2021-05-31Ａccept Date: 2021-06-02

Authors' contribution: All authors shared in the design, editing, and revision of the study.

Gabal HA and Gamal Eldin DA were responsible for data analysis.

\begin{abstract}
Introduction: Mental health problems are growing issues among college campuses. Improving adolescent mental well-being remains a challenge for most societies. Aim of work: To measure the prevalence of depression, anxiety, and stress among medical students and to identify the different risk factors associated with mood disorders among study participants. Materials and Methods: A cross-sectional study was conducted among a convenience sample of medical students at Ain Shams University, assessment of psychological mood disorders was done by using Depression, Anxiety, and Stress Scale-42 (DASS-42), alongside a pretested socio-demographic self-administered questionnaire. Results: A total of 240 students participated in the study with a mean age of $20.54 \pm 1.38$ years. The overall prevalence of depression, anxiety, and stress were $64.2 \%, 77.1 \%$, and 70.4\% respectively. Gender, Body Mass Index (BMI), physical activity and screen use affect the severity of depression while age, gender, and physical activity affect the severity of anxiety $(\mathrm{p}<0.05)$. Gender and physical activity as an entertainment method affect stress severity among studied students $(\mathrm{p}<0.05)$. By Multiple logistic regression analysis; age $(\mathrm{OR}=0.68, \mathrm{CI}=0.05-0.92)$, BMI $(\mathrm{OR}=2.58, \mathrm{CI}=1.32-5.05)$, being in clinical years $(\mathrm{OR}=3.90, \mathrm{CI}=1.49-10.17)$, and use screens for entertainment $(\mathrm{OR}=1.85, \mathrm{CI}=1.01-3.39)$ affect the occurrence of depression. Practicing physical activity $(\mathrm{OR}=0.48, \mathrm{CI}=0.23-0.99)$ was protective against anxiety
\end{abstract}


while screen use $(\mathrm{OR}=2.00, \mathrm{CI}=1.03-3.90)$ increase the risk for anxiety. Young age $(\mathrm{OR}=0.46, \mathrm{CI}=0.22-0.99)$, being female $(\mathrm{OR}=3.25, \mathrm{CI}=1.44-7.31)$, and using a screen for entertainment $(\mathrm{OR}=2.18, \mathrm{CI}=1.02-4.63)$ affect the occurrence of stress. Conclusion and recommendations: A substantial proportion of medical students are suffering from psychological mood disorders. Psychological intervention programs alongside adequate and appropriate students' supportive services should be carried out to decrease the burden of psychological mood disorders.

Keywords: Prevalence, Medical students, Depression, Stress and Anxiety

\section{Introduction}

Mental health problems are one of the world's major causes of disability according to the World Health Organization (WHO); accounting for $30.8 \%$ of total disability and $12.3 \%$ of the total burden of disease and this latter percent is expected to increase (WHO, 2019).

University students are a distinctive group who are at risk to have psychological problems, especially medical students, as they experience a crucial transitory phase through which they are transferred from puberty to adulthood being a difficult phase in a person's life (Ahmed et al., 2009 and Rotenstein et al., 2016).

High prevalence rates of psychological morbidity as depression and anxiety among medical students were reported by different studies, with psychological distress levels that were consistently higher than in the general population and age-matched peers (Kessler et al. 2007). Recent studies from Egypt, the United Arab Emirates, and Saudi Arabia have reported high prevalence of anxiety and depression among medical students (Abdel Wahed and Hassan, 2018).

Medical students are more vulnerable to psychological problems as they face various pressures and emotional difficulties during their transformation from a student to a young knowledgeable physician (Roopam et al., 2014); where possible sources of stress among medical students may include: academic stress, enormous syllabuses which should be covered within a short period, sudden changes in their style of learning, lack of adequate guidance, consideration of failure in exams, relationship with peers, parents' expectations, and changes in the educational medium (Ruprai et al., 2012).

Therefore, high rates of psychological mood disorders among medical students are most likely related to academic and social demands put on 
students by college environments when they are also involved in lifestyle and career issues (Jadoon et al., 2010).

In Egypt, mental disorders were associated with social, demographic, behavioral, and educational factors (Abdallah et al., 2014). Mood disorders can contribute to absenteeism and lack of confidence among medical students that will affect their performance (Roopam et al., 2014). Therefore, it is important to identify the prevalence, and risk factors for anxiety, stress, and depression among them.

\section{Aim of Work}

Tomeasure the prevalence ofanxiety, stress, and depression among medical students at Ain Shams University. Also, to identify different risk factors associated with psychological mood disorders among them.

\section{Material and Methods}

Study design: A cross-sectional study was conducted to achieve the objective of the study.

Study place and duration: The study was carried out for 3 months (from October to December 2020) among medical students at Ain Shams University in Cairo, Egypt. It was chosen because it is the third-oldest medical school in Egypt and enrols about 7000 students yearly (Ain Shams University Official home pages, 2021).

Study sample: A convenience sampling technique was used to collect data from medical students at the Faculty of Medicine, Ain Shams University. All students were eligible with the exclusion of first-year students as they do not have a history of academic performance. The Bachelor's of Medicine and Surgery program at the Faculty of Medicine is divided into two phases: a pre-clinical phase involving the first three years; and a clinical phase, which includes the last three years in the classic disciplinebased, and the last two years in the new fully integrated modular program (Ain Shams University Official home pages,2021).

Sample size calculated using Open Epi program, by reviewing results from a previous study (Fawzy and Hamed, 2017), showed that the prevalence of depression, anxiety, and stress among medical students were $(65 \%$, $73 \%$, 59.9\% respectively) using 90\% confidence interval and margin of error $5 \%$, based on these findings a sample size of 260 students were needed to achieve the study objective. Considering a $10 \%$ drop-out rate, the sample was exceeded 
to 286 participants. A response rate of $84 \%$ was achieved, so 240 students were included in the study.

Study methods: Anonymous, Arabic self-administrated questionnaire was used, the questionnaire was composed of:

Section 1: Socio-demographic data (e.g. age, sex, academic year, etc.), and socioeconomic status scale was used to assess the social class (ElGilany et al., 2012). It is an update of the old scoring system Fahmy and ElSherbini (1983). It has 7 domains with a total score of 84, which are the education and cultural, occupation, family, family possessions, economic, home sanitation, and health care domains. A higher score indicates better socioeconomic status. Total socioeconomic status was divided into four categories by quartiles as follows: Very low ( $\leq 25$ percentiles up to a score of 21), Low (25-50 percentiles i.e. a score of 22-42), Middle (50-75 percentiles i.e. a score of 43-63), High ( $\geq 75$ percentiles i.e. a score of 64 or higher).

Section2: Self-reported weight and height (BMI was calculated as weight in kilograms divided by the square of the height in meters $\left(\mathrm{kg} / \mathrm{m}^{2}\right)$ and was classified according to $\mathrm{WHO}$ classification: Underweight $(<18.5 \mathrm{~kg} /$ $\left.\mathrm{m}^{2}\right)$, Normal weight $(18.5-24.9 \mathrm{~kg} /$ $\mathrm{m} 2)$, Overweight (25-29.9 kg/m2), and Obese ( $\geq 30 \mathrm{~kg} / \mathrm{m} 2$ ) (WHO, 2018).

\section{Section3: Average academic} achievement which was categorized as Excellent ( $\geq 85 \%)$, Very good (75-84.9\%), Good (60-74.9\%), and Accepted $(<60 \%)$.

Section4: The Depression, Anxiety and Stress Scale (DASS) (Lovebird SH and Lovibond, 1995 and Moussa et al., 2017): It is a 42 items self-reported scale designed to measure the 3 negative emotional states of depression, anxiety, and stress. Each of the three DASS scales contains 14 items, divided into subscales of 2-5 items with similar content. The Depression scale assesses dysphoria, hopelessness, devaluation of life, self-deprecation, lack of interest/ involvement, anhedonia, and inertia. The Anxiety scale assesses autonomic arousal, skeletal muscle effects, situational anxiety, and subjective experience of anxious effect. The Stress scale is sensitive to levels of chronic non-specific arousal. It assesses difficulty relaxing, nervous arousal, and being easily upset/agitated, irritable/ over-reactive, and impatient. Subjects are asked to use 4-point severity/ 
frequency scales to rate the extent to which they have experienced each state over the past week. Scores for Depression, Anxiety, and Stress are calculated by summing the scores for the relevant items. Where scores are assigned according to: $\{$ score $=0\}$ Never, $\{$ score $=1\} \quad$ Sometimes, $\quad\{$ score $=2\}$ Often, $\{$ score $=3$ Almost always. The maximum score is 42 in each of the depression, anxiety, and stress scales. Lower scores are better.

-A pilot study was performed to cover $10 \%$ of the sample. The purpose was to establish the clarity of the tools. Also, it helped to estimate the time needed to fill the questionnaire. Pilot data were excluded from the results.

\section{Consent}

Informed consent was taken from students before starting the questionnaire and confidentiality was guaranteed through anonymity.

\section{Ethical Approval}

The ethical committee of the Faculty of Medicine, Ain Shams University, Egypt approved the research, and administrative approval was obtained.

\section{Data Management}

The data has been coded and registered and the analysis was done using the SPSS package (version 20). For qualitative variables, descriptive statistics were provided as frequencies and percentages, and as mean and standard deviation for quantitative variables. Chi-square and fissure exact were used to test the association between the degree of students' psychological morbidity and their age, gender, residence, BMI, and socioeconomic level. The correlation was used to test the relation between different psychological mood disorders, low correlation was when $r$ was between 0.10 to 0.29 , moderate when $r$ was between 0.30 and 0.49 , and high when $r$ was between 0.50 and 1.0 (Cohen et al., 2013). Multiple logistic regression analysis was used to identify the impact of different factors on students' psychological mood disorders (dependent variable was the presence or absence of depression, anxiety, or stress). $\mathrm{P}$-value $\leq 0.05$ was considered significant. 


\section{Results}

\section{Table (1): Sociodemographic and anthropometric characteristics of studied students $(\mathrm{No}=\mathbf{2 4 0})$ :}

\begin{tabular}{|c|c|c|c|}
\hline \multicolumn{3}{|l|}{ Characters } & No $(\%)$ \\
\hline Age & \multicolumn{2}{|r|}{$M e a n \pm S D(\min -\max )$} & $20.5418-24) 1.38 \pm)$ \\
\hline \multirow[t]{2}{*}{ Age category } & & $<20$ & $66(27.6 \%)$ \\
\hline & & $\geq 20$ & $173(72.4)$ \\
\hline Gender & & $\begin{array}{c}\text { Males } \\
\text { Females }\end{array}$ & $\begin{array}{c}92(38.3 \%) \\
148(61.7 \%)\end{array}$ \\
\hline Academic year & & $\begin{array}{c}\text { Preclinical } \\
\text { Clinical }\end{array}$ & $\begin{array}{c}159(66.3 \%) \\
81(33.8 \%)\end{array}$ \\
\hline $\begin{array}{l}\text { Average academic } \\
\text { achievement }\end{array}$ & & $\begin{array}{l}\text { Excellent } \\
\text { Very good } \\
\text { Good } \\
\text { Accepted }\end{array}$ & $\begin{array}{c}71(29.8 \%) \\
112(47.1 \%) \\
48(20.2 \%) \\
7(2.9 \%)\end{array}$ \\
\hline $\begin{array}{l}\text { Method of seeking } \\
\text { health information }\end{array}$ & & $\begin{array}{l}\text { Printed media } \\
\text { dal and hearing method } \\
\text { Both }\end{array}$ & $\begin{array}{c}87(37.5 \%) \\
132(56.9 \%) \\
13(5.6 \%) \\
\end{array}$ \\
\hline Residence & & $\begin{array}{c}\text { Urban } \\
\text { Rural } \\
\text { Slum area }\end{array}$ & $\begin{array}{c}203(84.6 \%) \\
28(11.7 \%) \\
9(3.7 \%)\end{array}$ \\
\hline Socioeconomic level & & $\begin{array}{l}\text { Very low } \\
\text { Low } \\
\text { Middle } \\
\text { High }\end{array}$ & $\begin{array}{c}0(0.0 \%) \\
13(5.4 \%) \\
101(42.1 \%) \\
126(52.5 \%) \\
\end{array}$ \\
\hline \multicolumn{3}{|c|}{ Socioeconomic level domains } & $M e a n \pm S D(\min -\max )$ \\
\hline \multicolumn{3}{|c|}{ Health Care domain } & $4.1 \pm 0.67(3-5)$ \\
\hline \multicolumn{3}{|c|}{ Family possessions domain } & 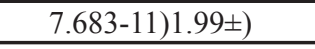 \\
\hline \multicolumn{3}{|c|}{ Education culture domain } & 23.651-29)5.32 \pm$)$ \\
\hline \multicolumn{3}{|c|}{ Occupation domain } & 7.101-10)3.08 \pm ) \\
\hline \multicolumn{3}{|c|}{ Family domain } & $7.454-10) 1.96 \pm)$ \\
\hline \multicolumn{3}{|c|}{ Home sanitation domain } & $8.305-12) 1.49 \pm)$ \\
\hline \multicolumn{3}{|c|}{ Economic domain } & $3.701-5) 0.79 \pm)$ \\
\hline \multirow{2}{*}{$\begin{array}{l}\text { Entertainment time } \\
\text { activities }\end{array}$} & Exercise & Yes & $66(27.5 \%)$ \\
\hline & $\begin{array}{l}\text { Screen } \\
\text { usage }^{\#}\end{array}$ & Yes & $144(60 \%)$ \\
\hline BMI & \multicolumn{2}{|c|}{$\operatorname{Mean} \pm S D(\min -\max )$} & $23.7 \pm 3.9(12.75-36.51)$ \\
\hline BMI categories & \multicolumn{2}{|c|}{$\begin{array}{c}\text { Underweight } \\
\text { Overweight } \\
\text { Obese }\end{array}$} & $\begin{array}{c}15(6.4 \%) \\
139(59.4 \%) \\
68(29.1 \%) \\
12(5.1 \%) \\
\end{array}$ \\
\hline
\end{tabular}

\#: Use of cell phones, laptops to reach internet and social media and use of TV screens

BMI: Body Mass Index. 
Table1 showed that 240 students participated in the study with a mean age of $20.54 \pm 1.38$ years, most of them were females $61.7 \%, 84.6 \%$ were urban residents, and more than half of participants (52.5\%) lived at high socioeconomic standard, $66.3 \%$ were studying in preclinical years and $47.1 \%$ had very good academic achievement. Students reported that $27.5 \%$ of them practiced physical activity and $60 \%$ used screens for entertainment in their spare time. The mean BMI of studied students was $23.7 \pm 3.9$, while $29.1 \%$ and $5.1 \%$ were overweight and obese respectively.

Figure (1) Severity of psychological mood disorders among the studied students:

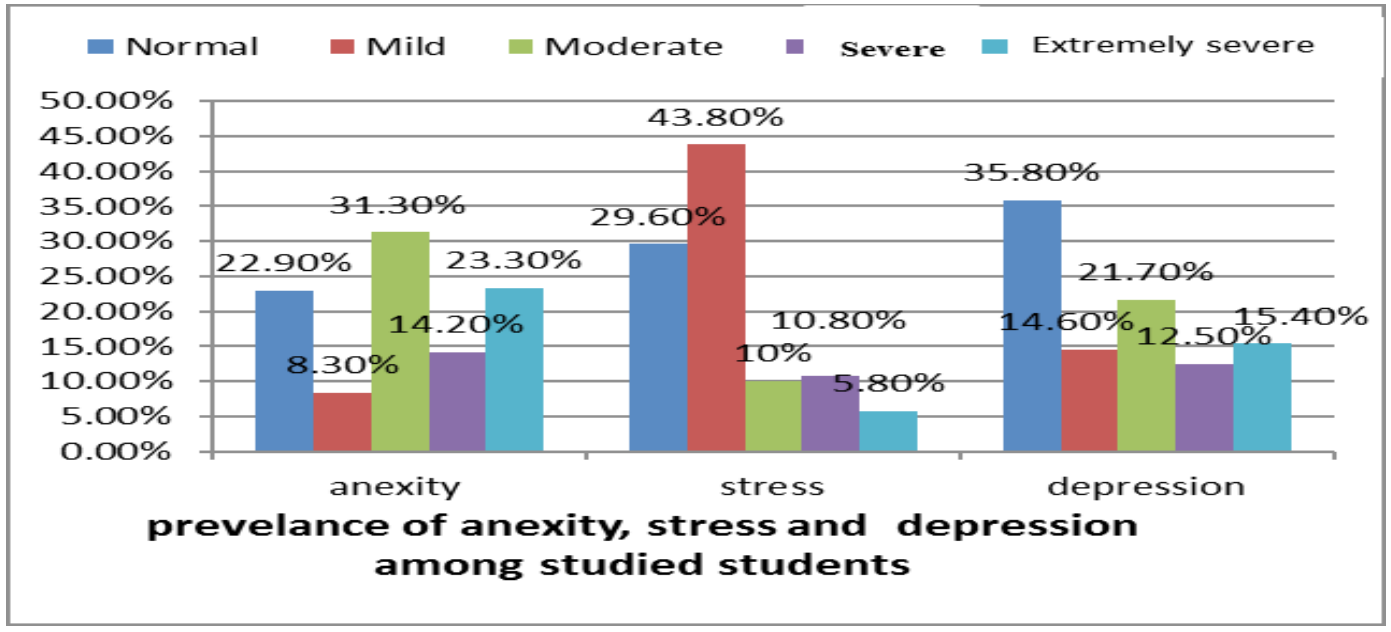

Figure1 represents the severity of psychological mood disorders; moderate depression and anxiety were reported by $21.70 \%$ and $31.30 \%$ of students respectively while $43.80 \%$ suffered from mild stress.

Mean depression, anxiety, and stress scores among studied students were $1510 \pm$ (0-42), $14 \pm 8(0-42)$, and $18 \pm 8(1-42)$ respectively. Regarding the prevalence of mood disorders $64.2 \%, 77.1 \%$, and $70.4 \%$ suffered from depression, anxiety, and stress respectively (results are not tabulated).

Depression score highly correlated to anxiety $(\mathrm{r}=0.66, \mathrm{p}$-value $=<0.001)$ and to stress score $(\mathrm{r}=0.63$, $\mathrm{p}$-value $=<0.001)$. Also, stress score highly correlated to anxiety score among studied students $(\mathrm{r}=0.61, \mathrm{p}$-value $=<0.001)$ (results are not tabulated). 
Table (2) Sociodemographic and anthropometric factors associated with degree of depression and anxiety among studied students:

\begin{tabular}{|c|c|c|c|c|c|c|}
\hline \multicolumn{3}{|l|}{ Characters } & $\begin{array}{l}\text { Normal } \\
\text { No }(\%)\end{array}$ & $\begin{array}{l}\text { Mild \& } \\
\text { Moderate } \\
\text { No }(\%)\end{array}$ & $\begin{array}{c}\text { Severe } \\
\text { \& very } \\
\text { severe } \\
\text { No }(\%)\end{array}$ & p value \\
\hline \multicolumn{7}{|l|}{ Depression } \\
\hline Age/ years & \multicolumn{2}{|l|}{$\begin{array}{l}<20 \\
\geq 20\end{array}$} & $\begin{array}{l}20(30.3) \\
66(38.2)\end{array}$ & $\begin{array}{l}24(36.4) \\
63(36.4)\end{array}$ & $\begin{array}{l}22(33.3) \\
44(25.4)\end{array}$ & $0.38^{\# \#}$ \\
\hline Gender & \multicolumn{2}{|l|}{$\begin{array}{l}\text { Male } \\
\text { Female }\end{array}$} & $\begin{array}{l}42(45.7) \\
44(29.7)\end{array}$ & $\begin{array}{l}27(29.3) \\
60(40.5)\end{array}$ & $\begin{array}{l}23(25.0) \\
44(29.7)\end{array}$ & $0.04^{\# \# *}$ \\
\hline $\begin{array}{l}\text { Socioeconomic } \\
\text { standard }\end{array}$ & \multicolumn{2}{|l|}{$\begin{array}{l}\text { low } \\
\text { Middle } \\
\text { High }\end{array}$} & $\begin{array}{l}5(38.5) \\
37(36.6) \\
44(34.9)\end{array}$ & $\begin{array}{c}5(38.5) \\
36(35.6) \\
46(36.5)\end{array}$ & $\begin{array}{c}3(23.1) \\
28(27.7) \\
36(28.6)\end{array}$ & $0.99^{\#}$ \\
\hline Residence & \multicolumn{2}{|c|}{$\begin{array}{c}\text { Urban } \\
\text { Rural \& Slum area }\end{array}$} & $\begin{array}{l}75(36.9) \\
11(29.7)\end{array}$ & $\begin{array}{c}73(36) \\
14(37.8)\end{array}$ & $\begin{array}{l}55(27.1) \\
12(32.4)\end{array}$ & $0.66^{\#}$ \\
\hline Academic years & \multicolumn{2}{|c|}{$\begin{array}{c}\text { Preclinical } \\
\text { Clinical }\end{array}$} & $\begin{array}{l}60(37.7) \\
26(32.1)\end{array}$ & $\begin{array}{l}54(34.0) \\
33(40.7)\end{array}$ & $\begin{array}{l}45(28.3) \\
22(27.2)\end{array}$ & $0.55^{\# \#}$ \\
\hline BMI & \multicolumn{2}{|c|}{$\begin{array}{c}\text { Underweight\& Normal } \\
\text { Overweight } \\
\text { Obese }\end{array}$} & $\begin{array}{l}67(43.5) \\
17(25.0) \\
2(16.7)\end{array}$ & $\begin{array}{c}52(33.8) \\
30(44.1) \\
2(16.7)\end{array}$ & $\begin{array}{c}35(22.7) \\
21(30.9) \\
8(66.7)\end{array}$ & $<0.001^{\# *}$ \\
\hline $\begin{array}{l}\text { Average academic } \\
\text { achievement }\end{array}$ & \multicolumn{2}{|c|}{$\begin{array}{l}\text { Excellent } \\
\text { Very good } \\
\text { Good } \\
\text { Accepted }\end{array}$} & $\begin{array}{c}28(39.4) \\
45(40.2) \\
10(20.8) \\
2(28.6)\end{array}$ & $\begin{array}{c}23(32.4) \\
35(31.2) \\
24(50.0) \\
4(57.1)\end{array}$ & $\begin{array}{c}20(28.2) \\
32(28.6) \\
14(29.2) \\
1(14.3)\end{array}$ & $0.67^{\#}$ \\
\hline \multirow{3}{*}{$\begin{array}{l}\text { Entertainment time } \\
\text { activities }\end{array}$} & Exercise & $\frac{\text { Yes }}{\text { NO }}$ & $\frac{32(48.5)}{54(31.0)}$ & $\frac{21(31.8)}{66(37.9)}$ & $\frac{13(19.7)}{54(31.0)}$ & $0.03^{\# *}$ \\
\hline & \multirow{2}{*}{ Screen usage } & Yes & $44(30.6)$ & $53(36.8)$ & $47(32.6)$ & \multirow{2}{*}{$0.05^{\# \#^{*}}$} \\
\hline & & NO & $42(43.8)$ & $34(35.4)$ & $20(20.8)$ & \\
\hline \multicolumn{7}{|l|}{ Anxiety } \\
\hline Age & $\begin{array}{l}<20 \\
\geq 20\end{array}$ & & $\begin{array}{l}15(22.7) \\
40(23.1)\end{array}$ & $\begin{array}{l}19(28.8) \\
76(43.9)\end{array}$ & $\begin{array}{l}32(48.5) \\
57(32.9)\end{array}$ & $0.05^{\# \# *}$ \\
\hline
\end{tabular}




\begin{tabular}{|c|c|c|c|c|c|c|}
\hline Gender & \multicolumn{2}{|l|}{$\begin{array}{l}\text { Male } \\
\text { Female }\end{array}$} & $\begin{array}{l}26(28.3 \%) \\
29(19.6 \%)\end{array}$ & $\begin{array}{l}43(46.7 \%) \\
52(35.1 \%)\end{array}$ & $\begin{array}{l}23(25.0 \%) \\
67(45.3 \%)\end{array}$ & $\mathbf{0 . 0 1}^{\# \#^{*}}$ \\
\hline $\begin{array}{l}\text { Socioeconomic } \\
\text { standard }\end{array}$ & \multicolumn{2}{|l|}{$\begin{array}{l}\text { low } \\
\text { Middle } \\
\text { High }\end{array}$} & $\begin{array}{l}2(15.4) \\
23(22.8) \\
30(23.8)\end{array}$ & $\begin{array}{l}7(53.8) \\
34(33.7) \\
54(42.9)\end{array}$ & $\begin{array}{c}4(30.8) \\
44(43.6) \\
42(33.3)\end{array}$ & $0.43^{\#}$ \\
\hline Residence & \multicolumn{2}{|c|}{$\begin{array}{c}\text { Urban } \\
\text { Rural\& Slum area }\end{array}$} & $\begin{array}{l}46(22.7) \\
9(24.3)\end{array}$ & $\begin{array}{l}81(39.9) \\
14(37.8)\end{array}$ & $\begin{array}{l}76(37.4) \\
14(37.8)\end{array}$ & $0.96^{\#}$ \\
\hline Academic years & \multicolumn{2}{|l|}{$\begin{array}{l}\text { Preclinical } \\
\text { Clinical }\end{array}$} & $\begin{array}{l}35(22.0) \\
20(24.7)\end{array}$ & $\begin{array}{l}67(42.1) \\
28(34.6)\end{array}$ & $\begin{array}{l}57(35.8) \\
33(40.7)\end{array}$ & $0.53^{\# \#}$ \\
\hline BMI & \multicolumn{2}{|c|}{$\begin{array}{c}\text { Underweight\& Normal } \\
\text { Overweight } \\
\text { Obese }\end{array}$} & $\begin{array}{l}41(26.6) \\
12(17.6) \\
2(16.7)\end{array}$ & $\begin{array}{c}64(41.6) \\
28(41.2) \\
3(25.0)\end{array}$ & $\begin{array}{c}49(31.8) \\
28(41.2) \\
7(58.3)\end{array}$ & $0.24^{\#}$ \\
\hline $\begin{array}{l}\text { Average academic } \\
\text { achievement }\end{array}$ & \multicolumn{2}{|l|}{$\begin{array}{l}\text { Excellent } \\
\text { Very good } \\
\text { Good } \\
\text { Accepted }\end{array}$} & $\begin{array}{c}19(26.8) \\
23(20.5) \\
8(16.7) \\
4(57.1)\end{array}$ & $\begin{array}{c}26(36.6) \\
48(42.9) \\
19(39.6) \\
1(14.3)\end{array}$ & $\begin{array}{c}26(36.6) \\
41(36.6) \\
21(43.8) \\
2(28.6)\end{array}$ & $0.35^{\#}$ \\
\hline \multirow{3}{*}{$\begin{array}{l}\text { Entertainment time } \\
\text { activities }\end{array}$} & Exercise & Yes & $23(34.8)$ & $\frac{27(40.9)}{68(39.1)}$ & $74(42.5)$ & $0.01^{\# \# *}$ \\
\hline & \multirow{2}{*}{ Screen usage } & Yes & $26(18.1)$ & $59(41.0)$ & $59(41.0)$ & \multirow{2}{*}{$0.08^{\# \#}$} \\
\hline & & $\mathrm{NO}$ & $29(30.2)$ & $36(37.5)$ & $31(32.3)$ & \\
\hline
\end{tabular}

BMI: Body Mass Index.

Table 2 showed that female students suffered from mild and moderate depression and from severe and extremely severe anxiety compared to males ( $p$-value $<0.05$ ). Overweight students suffered from mild and moderate depression while obese from severe and extremely severe depression. Students who didn't practice physical activity and used screen as a method for entertainment experienced mild and moderate depression compared to other students. Students aged less than 20 years suffered from severe and extremely severe anxiety compared to mild and moderate anxiety among students who aged 20 years or older. And students who didn't practice physical activity in their spare time experienced severe and extremely severe anxiety. 
Table (3): Sociodemographic and anthropometric factors associated with degree of stress among studied students:

\begin{tabular}{|c|c|c|c|c|c|c|}
\hline \multicolumn{3}{|l|}{ Stress } & $\begin{array}{l}\text { Normal } \\
\text { No }(\%)\end{array}$ & $\begin{array}{c}\text { Mild \& } \\
\text { Moderate } \\
\text { No }(\%)\end{array}$ & $\begin{array}{c}\text { Severe \& } \\
\text { very severe } \\
\text { No }(\%)\end{array}$ & $p$ value \\
\hline Age & \multicolumn{2}{|c|}{$\begin{array}{l}<20 \\
\geq 20\end{array}$} & $\begin{array}{l}\text { 22.7))15 } \\
32.4)) 56\end{array}$ & $\begin{array}{l}35(53.0) \\
93(53.8)\end{array}$ & $\begin{array}{l}16(24.2) \\
24(13.9)\end{array}$ & $0.10^{\# \#}$ \\
\hline Gender & \multicolumn{2}{|c|}{$\begin{array}{l}\text { Male } \\
\text { Female }\end{array}$} & $\begin{array}{l}42.4 \%)) 39 \\
21.6 \%) 32\end{array}$ & $\begin{array}{l}46.7 \%)) 43 \\
58.1 \%)) 86\end{array}$ & $\begin{array}{l}10.9 \%)) 10 \\
20.3 \%)) 30\end{array}$ & $<0.001^{\# \# *}$ \\
\hline $\begin{array}{l}\text { Socioeconomic } \\
\text { standard }\end{array}$ & \multicolumn{2}{|c|}{$\begin{array}{l}\text { low } \\
\text { Middle } \\
\text { High }\end{array}$} & $\begin{array}{l}23.1)) 3 \\
24.8)) 25 \\
34.1)) 43\end{array}$ & $\begin{array}{c}61.5)) 8 \\
55.4)) 56 \\
65(51.6)\end{array}$ & $\begin{array}{c}15.4)) 2 \\
20(19.8) \\
18(14.3)\end{array}$ & $0.53^{\#}$ \\
\hline Residence & \multicolumn{2}{|c|}{$\begin{array}{c}\text { Urban } \\
\text { Rural \& Slum area }\end{array}$} & $\begin{array}{l}30.0)) 61 \\
27.0)) 10\end{array}$ & $\begin{array}{c}54.7)) 111 \\
48.6)) 18\end{array}$ & $\begin{array}{l}15.3)) 31 \\
9(24.3)\end{array}$ & $0.39^{\#}$ \\
\hline Academic years & \multicolumn{2}{|c|}{$\begin{array}{l}\text { Preclinical } \\
\text { Clinical }\end{array}$} & $\begin{array}{l}\text { 29.6) } 47 \\
29.6)) 24\end{array}$ & $\begin{array}{l}50.3)) 80 \\
60.5)) 49\end{array}$ & $\begin{array}{l}32(20.1) \\
9.9)) 8\end{array}$ & $0.11^{\# \#}$ \\
\hline BMI & \multicolumn{2}{|c|}{$\begin{array}{c}\text { Underweight\& Normal } \\
\text { Overweight } \\
\text { Obese }\end{array}$} & $\begin{array}{l}32.5)) 50 \\
29.4)) 20 \\
8.3 \%) 1\end{array}$ & $\begin{array}{l}55.2)) 85 \\
47.1)) 32 \\
58.3 \%)) 7\end{array}$ & $\begin{array}{l}12.3)) 19 \\
23.5)) 16 \\
33.3 \%)) 4\end{array}$ & $0.06^{\#}$ \\
\hline $\begin{array}{l}\text { Average academic } \\
\text { achievement }\end{array}$ & \multicolumn{2}{|c|}{$\begin{array}{l}\text { Excellent } \\
\text { Very good } \\
\text { Good } \\
\text { Accepted }\end{array}$} & $\begin{array}{c}32.4 \%)) 23 \\
27.7 \%)) 31 \\
31.2 \%)) 15 \\
14.3 \%)) 1\end{array}$ & $\begin{array}{l}50.7 \%)) 36 \\
55.4 \%) 62 \\
56.2 \%)) 27 \\
42.9 \%)) 3\end{array}$ & $\begin{array}{l}16.9 \%)) 12 \\
17.0 \%) 19 \\
12.5 \%) 66 \\
42.9 \%)) 3\end{array}$ & $0.76^{\#}$ \\
\hline \multirow{4}{*}{$\begin{array}{l}\text { Entertainment time } \\
\text { activities }\end{array}$} & \multirow{2}{*}{ Exercise } & NO & $24.1 \%)) 42$ & $56.3 \%)) 98$ & $19.5 \%)) 34$ & \multirow{2}{*}{$0.01^{\# \# *}$} \\
\hline & & Yes & $43.9 \%)) 29$ & $47.0 \%)) 31$ & $9.1 \%) 6$ & \\
\hline & \multirow{2}{*}{ Screen time } & NO & $35.4 \%)) 34$ & $51.0 \%) 49$ & $13.5 \%)) 13$ & \multirow{2}{*}{$0.22^{\# \#}$} \\
\hline & & Yes & $25.7 \%)) 37$ & $55.6 \%)) 80$ & $18.8 \%)) 27$ & \\
\hline
\end{tabular}

$\# \#: \chi^{2}$ test,

\# : Fisher exact test,

*: Statistically significant at $\mathrm{p}$-value $\leq 0.05$

BMI: Body Mass Index.

Table 3 revealed that the degree of stress differed significantly according to gender; female student suffered from mild and moderate stress compared to males ( $p$-value $<0.05$ ). Students who didn't practice physical activity in their spare time experienced mild and moderate stress compared to other students practicing physical activity and the difference was statistically significant $(p<0.05)$. 
By studying the relation between different socioeconomic domains and mood disorders among studied students; low negative significant correlation was found between family domain and depression score $(r=-0.15$, $p$-value $=0.02)$, stress score $(\mathrm{r}=-0.18, \mathrm{p}$-value $=0.01)$ and anxiety score $(\mathrm{r}=-0.15$, $\mathrm{p}$-value $=0.03)$. No association was found concerning other domains (results are not tabulated).

Table (4): Factors affecting depression, anxiety and stress among studied students by binary logistic regression:

\begin{tabular}{|c|c|c|c|c|c|c|}
\hline \multirow{2}{*}{\multicolumn{2}{|c|}{ Characteristics }} & \multirow{2}{*}{ B } & \multirow{2}{*}{$\begin{array}{l}\text { Sig. } \\
\text { Lower }\end{array}$} & \multirow{2}{*}{$\begin{array}{c}\text { Adjusted } \\
\text { OR } \\
\text { Upper }\end{array}$} & \multicolumn{2}{|c|}{ 95\% C.I. OR } \\
\hline & & & & & & \\
\hline \multicolumn{7}{|l|}{ Depression } \\
\hline \multicolumn{2}{|l|}{ Constant } & 8.90 & 0.01 & ---- & ---- & ---- \\
\hline \multicolumn{2}{|l|}{ Age } & -0.39 & $0.01 *$ & 0.68 & 0.50 & 0.92 \\
\hline Gender & Female\# & 0.64 & 0.05 & 1.90 & 0.99 & 3.62 \\
\hline Academic years & clinical years\# & 1.36 & $0.01 *$ & 3.90 & 1.49 & 10.17 \\
\hline \multirow{3}{*}{ Body Mass Index } & $\begin{array}{l}\text { Underweight and } \\
\text { normal\#\# }\end{array}$ & ---- & $0.01 *$ & ---- & ---- & ---- \\
\hline & Overweight & 0.95 & $0.01 *$ & 2.58 & 1.32 & 5.05 \\
\hline & Obese & 1.27 & 0.13 & 3.55 & 0.69 & 18.23 \\
\hline \multirow{2}{*}{$\begin{array}{l}\text { Entertainment } \\
\text { time activities }\end{array}$} & Exercise\# & -0.55 & 0.11 & 0.57 & 0.29 & 1.12 \\
\hline & Screen usage \# & 0.62 & $0.04 *$ & 1.85 & 1.01 & 3.39 \\
\hline \multicolumn{7}{|l|}{ Anxiety } \\
\hline \multicolumn{2}{|l|}{ Constant } & -0.18 & 0.95 & --- & --- & --- \\
\hline \multicolumn{2}{|l|}{ Age } & 0.02 & 0.85 & 1.02 & 0.80 & 1.30 \\
\hline Gender & Female \# & 0.43 & 0.25 & 1.53 & 0.74 & 3.16 \\
\hline \multirow{2}{*}{ Residence } & $\begin{array}{l}\text { Rural \&Slum } \\
\text { area\# }\end{array}$ & -0.43 & 0.37 & 0.65 & 0.26 & 1.64 \\
\hline & Excellent\#\# & --- & 0.16 & --- & --- & --- \\
\hline \multirow{3}{*}{$\begin{array}{l}\text { Average } \\
\text { achievement }\end{array}$} & Very good & 0.45 & 0.23 & 1.56 & 0.76 & 3.23 \\
\hline & Good & 0.55 & 0.27 & 1.73 & 0.66 & 4.54 \\
\hline & Accepted & -1.20 & 0.16 & 0.30 & 0.06 & 1.60 \\
\hline
\end{tabular}




\begin{tabular}{|c|c|c|c|c|c|c|}
\hline \multirow{2}{*}{$\begin{array}{l}\text { Entertainment } \\
\text { time activities }\end{array}$} & Exercise \# & -0.74 & $0.04^{*}$ & 0.48 & 0.23 & 0.99 \\
\hline & Screen usage \# & 0.69 & $0.04^{*}$ & 2.00 & 1.03 & 3.90 \\
\hline \multicolumn{7}{|l|}{ Stress } \\
\hline \multicolumn{2}{|l|}{ Constant } & -0.41 & 0.51 & --- & ---- & ---- \\
\hline \multicolumn{2}{|l|}{ Age } & -0.78 & $0.05^{*}$ & 0.46 & 0.21 & 0.99 \\
\hline Gender & Female \# & 1.18 & $<0.001 *$ & 3.25 & 1.44 & 7.31 \\
\hline Residence & $\begin{array}{l}\text { Rural \&Slum } \\
\text { area\# }\end{array}$ & -0.06 & 0.91 & 0.94 & 0.35 & 2.56 \\
\hline \multirow{4}{*}{$\begin{array}{l}\text { Average } \\
\text { achievement }\end{array}$} & Excellent\#\# & ---- & 0.26 & ---- & ---- & ---- \\
\hline & Very good & 0.38 & 0.34 & 1.46 & 0.68 & 3.16 \\
\hline & Good & -0.52 & 0.31 & 0.60 & 0.22 & 1.63 \\
\hline & Accepted & 1.07 & 0.38 & 2.91 & 0.27 & 31.76 \\
\hline \multirow{2}{*}{$\begin{array}{l}\text { Entertainment } \\
\text { time activities }\end{array}$} & Exercise \# & -0.68 & 0.12 & 0.51 & 0.22 & 1.16 \\
\hline & Screen use\# & 0.78 & $0.04 *$ & 2.18 & 1.02 & 4.63 \\
\hline
\end{tabular}

\#\#: Reference group,

*: Statistically significant; $\mathrm{p}$-value $\leq 0.05$

\#: Reference groups are preclinical years, males, lived in urban areas, who didn't use screen, didn't practice physical activity,

Multiple logistic regression analysis was done to identify different factors that affect the occurrence (presence/absence) of depression, anxiety, and stress among studied students after adjustment of other variables (trying different models to reach the best model that can classify students appropriately). Age $(\mathrm{OR}=0.68, \mathrm{CI}=0.05-0.92)$, BMI $(\mathrm{OR}=2.58, \mathrm{CI}=1.32-5.05)$, being in clinical years $(\mathrm{OR}=3.90, \mathrm{CI}=1.49-10.17)$ and use screens for entertainment $(\mathrm{OR}=1.85, \mathrm{CI}=1.01$ 3.39) affect the occurrence of depression. Young, overweight students in clinical years who use screens for entertainment suffered more from depression compared to other students. Practicing physical activity $(\mathrm{OR}=0.48, \mathrm{CI}=0.23-0.99)$ was protective against anxiety while screen use $(\mathrm{OR}=2.00, \mathrm{CI}=1.03-3.90)$ increased the risk for anxiety. Young age $(\mathrm{OR}=0.46, \mathrm{CI}=0.22-0.99)$, female gender $(\mathrm{OR}=3.25$, $\mathrm{CI}=1.44-7.31)$, and using screens for entertainment $(\mathrm{OR}=2.18, \mathrm{CI}=1.02-4.63)$ affect the occurrence of stress (Table 4). 


\section{Discussion}

Psychological mood disorders are common illnesses worldwide that significantly impacted health and social life. Medical students are more prone to psychological mood disorders than the general public due to differences in the type of study, personal life, and social factors besides everyday stress. The objectives of the present study were to measure the prevalence of anxiety, stress, and depression among medical students at Ain Shams University and to identify the different risk factors associated with mood disorders among study participants.

Mean depression, anxiety, and stress scores among studied students were $15 \pm 10(0-42), 14 \pm 8(0-42)$, and $18 \pm 8(1-42)$ respectively. Regarding the prevalence of mood disorders were, $64.2 \%, 77.1 \%$ and $70.4 \%$ suffered from depression, anxiety, and stress respectively. Regarding the severity of psychological mood disorders: moderate depression and anxiety were reported by $21.70 \%$ and $31.30 \%$ of students respectively while $43.80 \%$ suffered from mild stress (Figure 1).

Similarly, Islam et al., 2020, surveyed 400 undergraduate students in Bangladesh using the GAD-7 scale and PHQ-9 to assess the prevalence of depression and anxiety and their potential associated factors, and they found that the prevalence rates of depression and anxiety were 69.5\% and $61 \%$ respectively. Another study was done in Bangladesh by Alim et al., 2017, among medical college students, which concluded a lower prevalence of depression (54.3\%) while the prevalence of anxiety was higher $64.8 \%$.

Lower rates were reported by a study done in Saudi Arabia to assess the traits of depression, anxiety, and stress among medical students in relation to potential underlying reasons using DASS-21. Prevalence of depression, anxiety, and stress were $43 \%, 63 \%$, and $41 \%$, respectively (Kulsoom and Afsar, 2015) while a study was done in Jizan University reported that the overall prevalence of stress was $71.9 \%$ (Sani et al., 2012).

An Egyptian study was performed among medical students at Menoufia University, detected that the prevalence of depression, anxiety, and stress were $63.3 \%, 78.4 \%$, and $57.8 \%$, respectively (Abdallah and Gabr, 2014). These findings agreed with the current study regarding depression and anxiety but disagreed regarding stress. 
Another study was done by Abolmagd et al.,2018, among 200 randomly chosen medical students using a semistructured interview of the Present State Examination. The results showed that $83 \%$ and $44 \%$ of students in the sample suffered from mood disorders and symptoms of anxiety respectively.

The current study findings agreed with Abdel Wahed and Hassan, 2017, who conducted a cross-sectional study among 442 medical students using DASS21 and they found that the prevalence of stress, anxiety, and depression was with various degrees and its' percent was $62.4 \%, 64.3 \%$, and $60.8 \%$ among the studied sample respectively. Also, a study conducted in a public university in Upper Egypt (Faculty of Medicine, Assiut University) and included 700 participants by Fawzy and Hamed, 2017, they measured the prevalence of depression, anxiety, and stress symptoms among medical students' participants by using a self-administered questionnaire, Depression Anxiety Stress Scale (DASS 21 ), and they revealed high percentages of depression (65\%), anxiety (73\%) and stress $(59.9 \%)$.

In a study which was done in Egypt among a group of first-year medical school students in Al Azhar University for Girls in Cairo using the Arabic short version of the standardized Depression Anxiety Stress Scale 42, the students of the Faculty of Medicine showed a high percentage of depression (96\%), anxiety (98\%), and stress $(76 \%)$ (Hammouda et al., 2020). They found that most of the students had moderate depression and stress (42\%, 36\% respectively), and $45 \%$ suffered from extreme anxiety. The variation between different studies may be attributed to the difference in the study population being restricted to firstyear students or preclinical and clinical students, in addition to the difference in the tools used for assessment.

Several studies concluded a high prevalence of mood problems among medical students other than agematched peers. Anxiety was the most prevalent mood disorder in medical students from the Middle East and Asia. About one in three medical students globally had anxiety; a prevalence rate which is higher than their peers in other educations. The global prevalence of anxiety in medical students' population was 33.8\% (95\% Confidence Interval: $29.2-38.7 \%$ ) while depression was $28.0 \% \quad(95 \%$ confidence interval; 24.2-32.1\%) (Puthran et al., 2016 and Quek et al.,2019) . This could be due to long studying years of Medicine 
and to the special nature of medical education curriculum in addition to the normal stressors of everyday life and consequently their social life affection.

Concerning the degree of mood disorders; depression, anxiety, and stress differed significantly according to gender, female students suffered from mild and moderate depression and stress but suffered from severe and extremely severe anxiety compared to males (Tables 2 and 3). Gender differences may be attributed to biological, sociocultural, or variable combinations of each. Female medical students are more competitive, tend to be more concerned about working hard to secure higher scores, and are more concerned about their performance (Amr et al., 2008).

In agreement with the current study, a study was done in Jizan University and found that females were more affected by stress than males $(77 \%)$ vs $(64 \%)$ and the difference was statistically significant $(\mathrm{p}<0.01$, odds ratio 1.89 CI 1.20 - 2.90) (Sani et al.,2012).

Similarly, Fawzy and Hamed, 2017 made univariate and multivariate statistical analysis and detected that females had higher scores of DASS compared to males. Meanwhile, an
Egyptian study done by Abdallah and Gabr, 2014 revealed that male university students were more prone to depression than female students, but females suffered more from anxiety compared to males.

On the other hand, Islam et al., 2020 , concluded that there were no significant gender differences in terms of depression and anxiety among studied students. Also, studies conducted by (Kulsoom and Afsar, 2015 and Alim et al., 2017) in Saudi Arabia and Bangladesh respectively concluded that there were no statistically significant gender differences found for depression or anxiety. Besides, subgroup analyses using gender which was conducted by (Quek et al., 2019), found no statistically significant differences in the prevalence of anxiety between males and females.

In the present work, multiple logistic regression analysis revealed that young overweight students in clinicalyears who used screens for entertainment suffered more from depression compared to other students. Practicing physical activity was protective against anxiety while screen use increases the risk for anxiety. Young age, female gender, and using the screen for entertainment affect the occurrence of stress (Table 
4). By studying different factors that might be associated with the degree of depression, anxiety and stress; BMI, practicing physical activity and screen use as a method for entertainment was associated with the increase in the severity of depression. Young-aged students who didn't practice physical activity in their spare time suffered from severe and extremely severe anxiety. Students who didn't practice physical activity in their spare time suffered from mild and moderate stress compared to other students and the difference was statistically significant (Table 2 and 3).

Similarly, Islam et al., 2020, found that there was no statistically significant association between sociodemographic variables (father's occupation and residence area) and mood disorders (depression or anxiety) but was associated with lack of physical activity, and excessive internet usage. But disagree with the current study regarding the effect of age.

Sani et al., 2012, found that sociodemographic variables were not statistically significantly associated with stress (as parents' level of education, occupation, and residence) while BMI was statistically significantly associated with stress.
On the other hand, (Hammouda et al., 2020) concluded that student's place of residence had a statistically significant effect on mood disorders (depression, anxiety, and stress), as students from rural areas suffered from mood disorders more than the urban resident. Another study from Egypt conducted by (Abdallah and Gabr, 2014) declared a statistically significant association between depression and socioeconomic variables as (place of residence and socio-economic status) as residents of rural areas and middleclass families were more likely to be depressed than urban and high social class, but agreed with the current study as there was no statistically significant association between anxiety and sociodemographic variables.

Abdel Wahed and Hassan, 2017 found that scores of stress and anxiety were statistically significantly associated with: female gender, increasing age and being obese. Higher depression score was statistically significantly associated with increasing age, low socioeconomic standard, and students residing in other governorates rather than Cairo. However, Fawzy and Hamed, 2017, detecded that students' residence area, being in preclinical years and students with lower academic 
achievement had higher scores of DASS compared to their intimates. The variation of the factors associated with psychological mood disorders could be explained by cultural and demographic variation from one place to another in addition to personal attributes.

The contemporary study showed that, depression score was highly correlated to anxiety $(\mathrm{r}=0.66$, $\mathrm{p}$-value $=<0.001)$ and to stress score $(\mathrm{r}=0.63, \quad \mathrm{p}$-value $=<0.001) . \quad$ Also, stress score was highly correlated to anxiety score among studied students $(\mathrm{r}=0.61, \mathrm{p}$-value $=<0.001)$ (results are not tabulated). Similarly, Fawzy and Hamed, 2017 detected significant correlations between stress with depression and anxiety $(\mathrm{p}=0.0001)$.

Study Limitations: One of the major disadvantages of cross-sectional study that it doesn't determine the causal relationship (which comes first the cause or the effect). Moreover, this study was conducted among students in a famous university in capital city, further multicenter studies involving undergraduate students from Upper and Lower Egypt, public and private medical schools should be conducted with consideration of the influence of different factors including academic, nonacademic and personality trait.

Conclusion: A substantial proportion of medical students suffered from depression, stress, and anxiety. Multiple logistic regression analysis revealed that age $(\mathrm{OR}=0.68, \mathrm{CI}=0.05$ 0.92), $\quad \mathrm{BMI}(\mathrm{OR}=2.58, \mathrm{CI}=1.32-5.05)$, being in clinical years $(\mathrm{OR}=3.90, \mathrm{CI}=1.49-10.17)$ and use screens for entertainment $(\mathrm{OR}=1.85$, $\mathrm{CI}=1.01-3.39$ ) affect the occurrence of depression. Practicing physical activity $\quad(\mathrm{OR}=0.48, \quad \mathrm{CI}=0.23-0.99)$ was protective against anxiety while screen use $(\mathrm{OR}=2.00, \mathrm{CI}=1.03-3.90)$ increase the risk for anxiety. Young age $(\mathrm{OR}=0.46, \mathrm{CI}=0.22-0.99)$, female gender $\quad(\mathrm{OR}=3.25, \quad \mathrm{CI}=1.44-7.31)$ and using screen for entertainment $(\mathrm{OR}=2.18, \quad \mathrm{CI}=1.02-4.63)$ affect the occurrence of stress.

Recommendations: Psychological intervention program alongside adequate and appropriate students' supportive services should be carried out to decrease the burden of psychological mood disorders.

\section{Funding}

There has been no significant financial support for this work.

\section{Conflict of interest}




\section{The authors stated that they have no} conflict of interest.

\section{Acknowledgment}

Authors would like to express their great appreciation to participants who generously shared their time and agreed to participate in this study.

\section{References}

1. Abdallah AR and Gabr HM (2014): Depression, anxiety and stress among first year medical students in an Egyptian public university. Int Res J Med Med Sci; 2(1):11-9.

2. Abdel Wahed WY and Hassan SK (2017): Prevalence and associated factors of stress, anxiety and depression among medical Fayoum University students. Alexandria J Med; 53:7784. Doi: 10.1016/j.ajme.2016.01.005.

3. Abolmagd S, Adel A, El Tabei D, Salah H, Emadeldin M, et al., (2018): Psychiatric morbidity among medical students: An Egyptian study. Egypt J Psychiatr; 39:48-51. Doi: 10.4103/ejpsy.ejpsy_42_17

4. Ahmed I, Banu H, Al-Fageer R and Al-Suwaidi $R$ (2009): Cognitive emotions: depression and anxiety in medical students and staff. J Crit Care; 24:1-7. Doi: 10.1016/j.jcrc.2009.06.003

5. Ain Shams University Official home pages (2021). Available at: http://med.asu.edu.eg/ home/en/.

6. Alim SAH, Rabbani MG, Karim E, Mullick MS, Mamun A, et al., (2017): Assessment of depression, anxiety and stress among first year MBBS students of a public medical college, Bangladesh. Bang J Psychiatry; 29(1): 23-9. Doi:org/10.3329/bjpsy. v29i1.32748

7. Amr M, El Gilany A and El-Hawary A (2008): Does gender predict medical students' stress in Mansoura, Egypt? Med Educ Online; 13:12 .Doi;10.3885/meo.2008.Res00273

8. Buchanan JL (2012): Prevention of depression in the college student Population. A review of the literature. Arch Psychiatric Nurs; 26(1):2142. Doi: 10.1016/j.apnu.2011.03.003

9. Cohen J, Cohen P, West SG and Aiken LS (2013): Applied multiple regression/correlation analysis for the behavioral sciences. Routledge, 3rd edition. Doi: 10.4324/9780203774441. Available at: https://books.google.com.eg/ books/about/Applied_Multiple_Regression Correlation.html?id=gkalyqTMXNEC\&redir $\mathrm{esc}=\mathrm{y}$

10. El-Gilany AA, El-Wehady A and El-Wasify $M$ (2012): Updating and validation of the socioeconomic status scale for health research in Egypt. East Mediterr Health J; 18 (9):962-8.

11. Fahmy $S$ and El-Sherbini AF (1983): Determining simple parameters for social classifications for health research. Bull High Inst Public Health; 13:95-108.

12. Fawzy M and Hamed AS (2017): Prevalence of psychological stress, depression and anxiety among medical students in Egypt. Psychiatry Res; 255:186-94. Doi: 10.1016/j. psychres.2017.05.027.

13. Hammouda SM, El Shafie TM and El Shennawy E (2020): The prevalence of depression, anxiety, and stress among a sample of first-year medical students. Egypt J Psychiatr; 41:111-6. Doi: 10.4103/ejpsy.ejpsy_3_20

14. Islam S, Akter R, Sikder T and Griffiths DM (2020): Prevalence and Factors Associated with Depression and Anxiety Among First-Year University Students in Bangladesh: A CrossSectional Study. Int J Ment Health Addiction. https://doi.org/10.1007/s11469-020-00242-y.

15. Jadoon NA, Yaqoob R, Raza A, Shehzad MA and Choudhry ZS (2010): Anxiety and depression among medical students: a cross-sectional study. J Pak Med Assoc; 60(8):699-702.

16. Kessler RC, Amminger GP, Aguilar-Gaxiola S, Alonso J, Lee S, et al., (2007): Age of onset of mental disorders: a review of recent literature. Curr Opin Psych; 20:359-64. Doi: 10.1097/ 
YCO.0b013e32816ebc8c

17. Kulsoom B and Afsar NA (2015): Stress, anxiety, and depression among medical students in a multi-ethnic setting. Neuropsychiatr Dis Treat; 11:1713-22. Doi: 10.2147/NDT.S83577. PMID: 26213470; PMCID: PMC4509544

18. Lovebird SH and Lovibond (1995): Manual for the depression anxiety stress scales. 2nd. Ed. Sydney: Psychology Foundation. Available at https://researchingparents.files.wordpress. com/2013/02/dass.pdf

19. Moussa MT, Lovibond PF, Laube R and Megahead HA (2017): Psychometric properties of an Arabic version of the Depression Anxiety Stress Scales (DASS21). Res Soc Work Pract; 27(3):375-86. Doi:10.1177/1049731516662916

20. Puthran R, Zhang MW, Tam WW and Ho RC (2016): Prevalence of depression amongst medical students: a meta-analysis. Med Educ; 50(4):456-68. Doi: 10.1111/medu.12962.

21. Quek TT, Tam WW, Tran BX, Zhang M, Zhang Z, et al., (2019): The Global Prevalence of Anxiety Among Medical Students: A MetaAnalysis. Int J Environ Res public health;16 (15): 2735. Doi:10.3390/ijerph16152735

22. Roopam B, Saurabh S and Manjeet K (2014): A Study of Correlation of Anxiety Levels with Body Mass Index in New Mbbs Students. Natl J Physiol Pharm Pharmacol; 4 (3): 208 - 12.
Doi:10.5455/njppp.2014.4.080420142

23. Rotenstein LS, Ramos MA, Torre M, Segal JB, Peluso MJ, et al., (2016): Prevalence of depression, depressive symptoms, suicidal ideation among medical students: a systematic review meta-analysis. JAMA; 316:2214-36. Doi:10.1001/jama.2016.17324

24. Ruprai RK, Kamble P and Kurwale M (2012): Environmental Stress and Changes in Anxiety Score and Reaction Time: A Comparative Study Among Day Scholars and Hostilities in First Year MBBS Students. Glob J Interdiscip Soc Sci; 1:1-3.

25. Sani M, Mahfouz MS, Bani I, Alsomily AH, Alagi D, et al., (2012): Prevalence of stress among medical students in Jizan University, Kingdom of Saudi Arabia. Gulf Med J; 1 (1):19-25

26. World Health Organization (2018): Obesity and overweight. Geneva: World Health Organization. Available at: https://www.who. int/news-room/fact-sheets/detail/ obesity-andoverweight.

27. World Health Organization (2019): The WHO Special Initiative for Mental Health (20192023): Universal Health Coverage for Mental Health. Available at: https://www.who.int/ publications-detail-redirect/special-initiativefor-mental-health-(2019-2023). 
